\title{
Involved margins after lumpectomy for breast cancer: always to be re-excised?
}

Luca Sorrentino $^{\mathrm{a}}$, MD, Manuela Agozzino ${ }^{\mathrm{b}}$, MD, Sara Albasini ${ }^{\mathrm{c}}$, MSc, Daniela Bossi ${ }^{\mathrm{c}}$, MD, Serena Mazzucchelli ${ }^{\mathrm{a}}, \mathrm{PhD}$, Renzo Vanna ${ }^{\mathrm{d}}, \mathrm{PhD}$, Ourania Papadopoulou ${ }^{\mathrm{e}}$, MD, Laura Villani ${ }^{\mathrm{b}}$, MD, Fabio Corsi $^{\mathrm{a}^{*}}, \mathrm{MD}$

\author{
aa Department of Biomedical and Clinical Sciences “Luigi Sacco", University of Milan, via G. B. Grassi 74, 20157 Milan, Italy \\ ${ }^{\text {b} P a t h o l o g y ~ U n i t, ~ I s t i t u t i ~ C l i n i c i ~ S c i e n t i f i c i ~ M a u g e r i ~ I R C C S, ~ v i a ~ S . ~ M a u g e r i ~ 10, ~} 27100$ Pavia, Italy \\ ${ }^{\mathrm{c}}$ Surgery Department, Breast Unit, Istituti Clinici Scientifici Maugeri IRCCS, via S. Maugeri 10, 27100 Pavia, Italy \\ ${ }^{\mathrm{d} N a n o m e d i c i n e ~ a n d ~ M o l e c u l a r ~ I m a g i n g ~ L a b, ~ I s t i t u t i ~ C l i n i c i ~ S c i e n t i f i c i ~ M a u g e r i ~ I R C C S, ~ v i a ~ S . ~ M a u g e r i ~ 10, ~ P a v i a, ~ I t a l y ~}$ \\ ${ }^{\text {e}}$ Service of Breast Radiology, Department of Radiology, Istituti Clinici Scientifici Maugeri IRCCS, via. S. Maugeri 10, Pavia, Italy
}

*Corresponding Authors:

Prof. Fabio Corsi, MD

Department of Biomedical and Clinical Sciences "Luigi Sacco", University of Milan, Via G. B. Grassi, 74, 20157 Milan, Italy

Surgery Department, Breast Unit, ICS Maugeri S.p.A. SB, via Maugeri 10, 27100 Pavia, Italy

E-mail: fabio.corsi@ unimi.it - Phone: +39.02.5031.9850 - Fax: +39.02.5031.9846

Funding: This research did not receive any specific grant from funding agencies in the public, commercial, or not-for-profit sectors.

Running Title: Avoiding re-excision of positive margins 


\section{Abstract}

Background: The oncologic benefit of upfront re-excision of involved margins after breastconserving surgery in the context of current multimodal clinical management of breast cancer is unclear. The aim of the present study was to assess the 5-years locoregional recurrence (LRR)-free and distant metastases (DM)-free survival probabilities in patients not undergoing re-excision of positive margins after lumpectomy for breast cancer.

Methods: A cohort of 104 patients with positive margins not undergoing re-excision was matched by propensity score with a cohort of 2,006 control patients with clear margins after breastconserving surgery, treated between 2008 and 2018. A multivariate survival analysis was performed accounting for all variables related to LRR and DM, including adjuvant treatments.

Results: After adjusting for potential confounders, avoiding to re-excise a positive margin after lumpectomy had no effect on 5-years LRR-free survival probability (HR 0.98, 95\%CI 0.36-2.67, $\mathrm{p}=0.96$ ) or 5-years DM-free survival probability (HR 0.37, 95\%CI 0.08-1.61, p=0.18). No correlation was found between occurrence of LRR and number of involved margins (HR 1.28, 95\%CI 0.10-12.4, Log-rank p=0.83), or extension of infiltrating disease (HR 1.21, 95\%CI 0.207.40, Log-rank $\mathrm{p}=0.83$ ), but a trend toward higher LRR probability was found for invasive ductal (HR 6.92, 95\%CI 0.7-68.8, Log-rank p=0.10) and invasive lobular cancer (HR 12.95, 95\%CI 0.79213.6, Log-rank $\mathrm{p}=0.07$ ) on positive margins.

Conclusions: In the era of multimodal treatment of breast cancer and accurate strategies to reduce the probability of residual disease in the post-lumpectomy cavity, re-excision of positive margins might be omitted in selected patients with low-risk breast cancers.

\section{Keywords}

Re-excision; Breast-conserving surgery; Breast cancer; Margins. 


\section{Introduction}

Lumpectomy with a single line of normal cells between cancer and the resection limit is currently the standard of care of early breast cancer [1]. Indeed, the great majority of updated guidelines clearly state that "no ink on tumor" is the standard adequate margin and involved margins are considered inacceptable [2, 3]. Positive margins are related to a higher loco-regional recurrence (LRR) rate, because residual disease is likely to be left in the surrounding tissues [4-6]. But histopathology on re-excisions not always reveals residual disease [7-9]. Furthermore, up to 20$50 \%$ of breast cancer patients treated by lumpectomy undergo re-excision of an involved margin and but re-excision is associated to heavier psychological burden for patients, delay of multidisciplinary case discussion and onset of adjuvant treatments, and increased costs [10, 11]. The benefit of upfront re-excision in all cases with involved margins in terms of LRR in current multimodal clinical treatment of breast cancer is not clear, since updated evidences are lacking [12]. The aim of the present study was to compare the 5-years LRR-free and distant metastases (DM)free survival probabilities between patients with involved margins not re-excised vs. patients with clear margins after breast-conserving surgery. 


\section{Material and methods}

\section{Study population}

Patients were retrospectively collected from the prospectively-maintained database of the EUSOMA-accredited Breast Unit of ICS Maugeri Hospital from January 2008 to January 2018. Inclusion criteria were proven diagnosis of breast cancer, any size if suitable for lumpectomy and evaluation of margins status on final histopathology. Exclusion criteria were: benign disease, indication for total mastectomy, neoadjuvant treatment, distant metastases at diagnosis, or palliative surgery without curative intent. A total of 2,160 patients affected by breast cancer and treated by breast conserving-surgery were reviewed.

\section{Surgical procedure}

All patients underwent preoperative radio-guided occult lesion localization. Briefly, 15-25 MBq of 99mTc-labelled human serum albumin nanocolloids (Nanocoll, GEHC, Italy) in $0.2 \mathrm{~mL}$ saline were injected by ultrasound guidance the day before surgery. A scintigraphy was performed to assess the adequacy of procedure. During surgery, lesion projection on breast skin was localized by a gamma probe (Bluetooth Neoprobe Gamma Detection System) to plan the incision. The excision was continuously guided by the gamma probe, to check the inclusion of the lesion within the specimen. After lesion resection, the gamma probe was used to assess possible residual signal in the cavity. In all cases, the sentinel lymph node biopsy was performed by radioisotope technique. All the procedures were performed by the same surgical equipe.

\section{Evaluation of margins and multidisciplinary management}

In all cases margins status was available on final pathology, and all patients were discussed in the weekly multidisciplinary meeting of our institution to plan the adjuvant treatments. The margins status was determined on all six planes of the surgical specimen. According to current guidelines, adequate margins were defined as "no ink on tumor" [2]. A focally positive margin was defined as 
cancer invading for less than $4 \mathrm{~mm}$ in length the inked margin; in case of tumor involvement of more than $4 \mathrm{~mm}$ in length on inked margin, this was considered as extensively involved. In case of positive margins, a re-intervention was considered mandatory: a re-excision was proposed in case of extensive involvement of a single margin or multiple focally positive margins, while total mastectomy was preferred for multiple extensive margins or if a misdiagnosed multifocal disease was suggested on histopathology after lumpectomy. Patients who refused the proposal of reexcision despite positive margins were the target population of the study.

\section{Study design and endpoints}

Patients were divided in two groups: those with negative margins at first surgery or undergoing a re-intervention after positive margins (control patients, $\mathrm{CP}$ ) and those with positive margins who were not subjected to re-excision (not re-excised, NR). The two groups were compared in terms of 1) 5-years LRR-free survival probability and 2) 5-years DM-free survival probability. LRR was defined as the occurrence of ipsilateral breast cancer or nodal disease at axillary, internal mammary and/or supraclavicular level, proven on core biopsy. DM was defined as the occurrence of distant lesions with computed tomography and positron emission tomography features suggestive of malignancy. We also evaluated: 1) cancer-related death rate, 2) impact of number of positive margins, extension and histology of infiltrating disease on LRR. Due to the non-random design of the study, a propensity score-matched analysis was performed matching NR and CP, to reduce bias resulting from possible confounders between the two groups. NR and $\mathrm{CP}$ were matched at 1:5 ratio according to the subsequent preoperative variables: age, histological type, $\mathrm{pT}$ and $\mathrm{pN}$ stages, grading and biomolecular subtype (Luminal A, Luminal B, HER2-positive and triple-negative). Propensity score matching was preferred given the presence of confounding variables which could affect the choice of treatment (re-excise or not re-excise), to account for this selection bias. However, since propensity score matching could decrease the available sample size, potentially under-powering the study, comparison between groups was also checked by re-analyzing the study 
population through inverse probability weights (IPW) adjustment, thus including all 2,160 patients. A multivariate survival analysis was performed accounting for all variables related to LRR and DM, including adjuvant treatments.

\section{Statistical analysis}

Differences between NR and CP after propensity score-matched analysis were assessed to verify the heterogeneity of the study population. Variables were reported as means \pm standard deviations or as absolute numbers and percentages. Categorical variables were compared using $\chi^{2}$ test or Fisher exact test as appropriate, while continuous variables were compared using Student's T test or nonparametric Wilcoxon test. To verify the adequacy of sample size to evaluate the end-points, the ideal sample size was calculated as for an equivalence study model. The null hypothesis was that reexcising or not re-excising a positive margin would have resulted in different outcomes in terms of disease free-survival. Considering a global recurrence rate of about $10 \%$ both for invasive cancer and ductal carcinoma in situ (DCIS), and a hypothesized difference between groups of 10-12\%, it would have been sufficient to include about 100 patients per group to achieve a study power of $90 \%$ with a confidence level equal to 0.05 . Based on these considerations, sample size was considered adequate. NR and $\mathrm{CP}$ were compared by Cox proportional hazard regression model including variables significantly associated with the outcomes, included type of adjuvant treatment. The 5years LRR-free and DM-free survival probabilities were estimated by the Kaplan-Meier method. Statistical significance was set at $\mathrm{p}<0.05$ (two tailed). Data analysis was performed using SAS software (v. 9.4, SAS Institute Inc., Cary, USA). 


\section{Results}

Margins status and distribution of baseline variables after propensity score-matching

Of 2,160 patients reviewed, 2,006 cases (92.9\%) had clear margins on final pathology after lumpectomy, while in 154 patients $(7.1 \%)$ positive margins were found. Of the latter 154 patients, $50(2.3 \%)$ were re-excised while $104(4.8 \%)$ refused re-intervention. Considering re-excised patients, in 18 cases $(36.0 \%)$ residual disease was found on histopathology after re-intervention, while no evidence of further cancer was encountered in the remaining 32 cases $(64.0 \%)$. After propensity score matching, a total of 624 patients were included for analysis of the endpoints: 104 NR (16.7\%) vs. 520 CP (83.3\%). Distribution of baseline variables was balanced between groups, as reported in Table 1. Radiotherapy was offered to $89.4 \%$ of NR vs. $81.4 \%$ of CP ( $\mathrm{p}=0.05$ ). Radiotherapy was delivered with a conventional 3D conformal technique to the whole breast with two tangential fields, for a total dose of 45 Gy in 20 fractions plus a possible boost of 5 Gy in 4 weekly fractions. Boost was delivered in $90.3 \%$ of CP vs. $95.7 \%$ of NR patients $(\mathrm{p}=0.11)$. Chemotherapy was administered respectively in $22.1 \%$ and $21.3 \%$ of cases $(\mathrm{p}=0.86)$. Chemotherapy regimen was based on anthracyclines with or without taxanes in $58.3 \%$ of NR vs. $45.5 \%$ of $\mathrm{CP}$, and trastuzumab was administered respectively in $38.9 \%$ and $45.5 \%$ of cases; in the remaining cases, other regimens $(2.8 \%$ vs. $9.0 \%)$ were offered $(\mathrm{p}=0.49)$. Hormone therapy was received by $73.1 \%$ of $\mathrm{NR}$ and $77.9 \%$ of $\mathrm{CP}(\mathrm{p}=0.29)$.

Long-term outcomes and event-free survival probabilities between not re-excised vs. control patients

Mean follow up after breast cancer surgery was $48.6( \pm 30.6)$ months in NR patients vs. $44.8( \pm 36.8)$ months in $\mathrm{CP}(\mathrm{p}=0.09)$. LRR occurred in $4.8 \%$ of NR patients and in $5.6 \%$ of $\mathrm{CP}(\mathrm{p}=0.75)$, while DM were observed respectively in $1.9 \%$ and $5.6 \%$ of cases $(\mathrm{p}=0.12)$. Of $34 \mathrm{LRR}, 23(67.6 \%)$ occurred as in-breast local disease, and 11 (32.4\%) as ipsilateral axillary and/or supra-clavicular nodal recurrence. No difference was observed also in cancer-related death rates, being $3.9 \%$ both in 
NR and CP $(p=1.00)$. All these data are reported in Table 2. NR and CP showed comparable 5-years LRR-free survival probability curves, with a hazard ratio (HR) for not re-excising a positive margin equal to $0.90(95 \%$ CI 0.35-2.35, Log-Rank test $\mathrm{p}=0.83)$, see Figure 1a. Also 5-years DM-free survival probabilities were similar between NR and CP (HR 0.33, 95\%CI 0.08-1.38, Log-Rank test $\mathrm{p}=0.11$ ), as showed in Figure 1b. After adjusting Kaplan-Meier survival analyses by IPW, not reexcising a positive margin still had no impact on 5-years LRR-free (Log-Rank test p=0.94) or DMfree (Log-Rank test p=0.14) survival probabilities, as showed in Supplementary Figures S1 and S2.

Multivariate survival analysis of not re-excising positive margins on LRR-free and DM-free survival probabilities

After adjusting for potential confounding variables by Cox regression model, avoiding to re-excise a positive margin after lumpectomy confirmed no effect on 5-years LRR-free survival probability, with an adjusted HR equal to 0.98 (95\% CI 0.36-2.67, $\mathrm{p}=0.96)$. No variable resulted to be independently associated to LRR. Not re-excising positive margins had no impact also on 5-years DM-free survival probability (adjusted HR 0.37, 95\%CI 0.08-1.61, p=0.18). Independent predictors of DM-free survival probability were nodal status (HR 0.22 for N1 vs. N2, 95\%CI 0.07-0.72, $\mathrm{p}=0.013$ ) and the need for adjuvant chemotherapy (HR 6.12,95\%CI 1.97-18.99, p=0.002). Cox multivariate analysis is reported on Table 3.

Impact of number of positive margins, extension and histopathology of infiltrating disease on LRR

In 79 NR patients (76.0\%) 1 single involved margins was found on final pathology, and 25 cases (24.0\%) had $>1$ positive margin. A focal involvement was encountered in 70 cases $(67.3 \%)$, while extensive infiltration was observed in 34 patients $(32.7 \%)$. No correlation was found between occurrence of LRR and number of involved margins (HR 1.28 for $>1$ vs. 1 involved margin, 95\%CI 0.10-12.4, Log-rank $\mathrm{p}=0.83$ ), or extension of infiltrating disease (HR 1.21 for extensive vs. focal infiltration, 95\%CI 0.20-7.40, Log-rank p=0.83). Histopathology on involved margins revealed 
DCIS in $68(65.4 \%)$ patients, ductal invasive cancer in $27(26.0 \%)$ and lobular invasive cancer in 9 (8.6\%) cases. No correlation was observed with LRR, with HR equal to 6.92 for invasive ductal vs DCIS (95\%CI 0.7-68.8, Log-rank p=0.10) and 12.95 for invasive lobular vs. DCIS (95\%CI 0.79213.6, Log-rank p=0.07). 


\section{Discussion}

An accurate preoperative lesion localization and the adoption of current guidelines on the adequacy of surgical margin in breast-conserving surgery have greatly reduced the re-excision rate and related healthcare costs $[10,11]$. Since several features of the proposed toolbox for reducing re-operations after breast cancer surgery are adopted in our institution, it is not surprising that only $2.3 \%$ of patients were re-excised to achieve adequate margins. Notably, in $4.8 \%$ of patients with involved margins a re-excision was refused. This is a major point of controversy, because currently no guideline accepts less than no ink on tumor for invasive cancer, with the only exception of Dutch guidelines which support the avoidance of re-intervention in case of focally positive margins [13].

It should be noted that the great majority of NR patients had pT1 $(72.1 \%)$, node-negative (75.0\%), Luminal A (56.0\%) lesions, therefore a large proportion of these patients had a low-risk breast cancer. Interestingly, chemotherapy $(22.1 \%$ vs. $21.3 \%, \mathrm{p}=0.86)$ and hormone therapy $(73.1 \%$ vs. $77.9 \%, \mathrm{p}=0.29)$ rates were comparable between $\mathrm{NR}$ patients and $\mathrm{CP}$, therefore omission of reexcision of positive margins was not associated to enhancement of adjuvant treatments. Conversely, a slight but not significant trend in a higher use of radiotherapy was evident in NR patients $(89.4 \%$ vs. $81.4 \%, \mathrm{p}=0.05)$, expectedly to minimize LRR occurrence.

After controlling for baseline features by propensity score matching, omitting to re-excise a positive margin was not related to a higher LRR rate, being $4.8 \%$ vs. $5.6 \%$ in control group $(p=0.75)$. Also, $\mathrm{DM}$ rates were similar $(\mathrm{p}=0.12)$. At multivariate analysis accounting also for post-operative treatments, included radiotherapy, still avoiding reoperation for positive margins had no effect on LRR (HR 0.98, p=0.96) or DM (HR 0.37, p=0.18). The absence of any effect on 5-years survival probabilities omitting to re-excise a positive margin is puzzling, but some explanations may be hypothesized. First, all patients underwent preoperative localization, therefore residual disease was unlikely to be left in post-lumpectomy cavity, as demonstrated by the fact that $64 \%$ of re-excised 
patients had not residual disease on specimen after re-operation. Several other studies have showed that often residual cancer is not found on final pathology after re-excision for a positive margin [7$9,14,15]$. In other words, a margin could be incorrectly considered positive after handling of the specimen for histopathological analysis, since the pathologist might misunderstand its interpretation due to specimen shrinkage $[16,17]$. Another point to be considered is the type of margin involvement. In the great majority of NR patients, a single $(76.0 \%)$, focally positive $(67.3 \%)$ margin involved by intraductal cancer $(65.4 \%)$ was observed on histopathology. Therefore, the eventuality of a clinically significant residual disease after lumpectomy is unlikely, and radiotherapy together with adjuvant treatments could have been sufficient to control the disease at loco-regional level [18].

Due to the low event rate, the association between histopathology of cancer at inked margin, extension of involvement, number of involved margins and LRR could not be analyzed. However, a non-significant trend in increasing LRR after omitting re-excision was observed for invasive ductal (HR 6.92, $\mathrm{p}=0.10$ ) and invasive lobular cancer (HR 12.95, $\mathrm{p}=0.07$ ) compared to DCIS.

Only few studies analyzing oncologic outcomes after not re-excising a positive margin after breastconserving surgery are available in recent literature [18-21]. Since Dutch breast cancer guidelines do not recommend re-excision for focally positive margins, Vos et al. published a study on 492 patients who were not subjected to re-intervention. LRR rate was $2.9 \%$ vs. $1.1 \%$ in case of reexcision, but the difference was not statistically significant (0.099), and no association was found with overall or disease-free survival [18]. Similarly, a study from the United States reported that in selected cases omitting re-excision of positive margins are not associated with increased LRR rates, particularly for anterior and posterior margins, if radiotherapy is administered [19]. Another study analyzing a large cohort proposed radiotherapy without re-excision as a viable option to control for LRR after positive margins on lumpectomy, in case of older patients with low-risk cancer [20]. 
Furthermore, the effect of radiotherapy could be maximized by boost delivered at the positive margin [21]. A recent meta-analysis confirmed that positive margins after lumpectomy predict a higher LRR (OR 1.96, $\mathrm{p}<0.001)$ [22], but it should be noted that most of reviewed evidences were published in another era of breast cancer treatment, when the definition of adequate margins and the availability of adjuvant treatments were quite different from current clinical practice [23].

\section{Conclusions}

In the era of multimodal treatment of breast cancer and accurate strategies to reduce the probability of residual disease in the post-lumpectomy cavity after breast-conserving surgery, re-excision for positive margins might be omitted in selected patients with low-risk breast cancers. Since a single focally positive margin is encountered in the majority of cases with involved margins and considering the absence of residual disease in most re-excised patients, avoiding re-operation could be discussed in such cases. 


\section{References}

1. Buchholz TA, Somerfield MR, Griggs JJ, et al. Margins for breast-conserving surgery with whole-breast irradiation in stage I and II invasive breast cancer: American Society of Clinical Oncology endorsement of the Society of Surgical Oncology/American Society for Radiation Oncology consensus guideline. J Clin Oncol 2014; 32(14):1502-6.

2. Moran MS, Schnitt SJ, Giuliano AE, et al. Society of Surgical Oncology-American Society for Radiation Oncology consensus guideline on margins for breast-conserving surgery with whole-breast irradiation in stages I and II invasive breast cancer. Ann Surg Oncol 2014; 21(3):704-16.

3. Coates AS, Winer EP, Goldhirsch A, et al. Tailoring therapies - improving the management of early breast cancer: St Gallen International Expert Consensus on the Primary Therapy of Early Breast Cancer 2015. Ann Oncol 2015; 26(8):1533-46.

4. Park CC, Mitsumori M, Nixon A, et al. Outcome at 8 years after breast-conserving surgery and radiation therapy for invasive breast cancer: influence of margin status and systemic therapy on local recurrence. J Clin Oncol 2000; 18(8):1668-75.

5. Bodilsen A, Bjerre K, Offersen BV, Vahl P, Ejlertsen B, Overgaard J, Christiansen P. The Influence of Repeat Surgery and Residual Disease on Recurrence After BreastConserving Surgery: A Danish Breast Cancer Cooperative Group Study. Ann Surg Oncol 2015; 22 Suppl 3:S476-85.

6. Kahlert S, Kolben TM, Schmoeckel E, et al. Prognostic impact of residual disease in simultaneous additional excision specimens after one-step breast conserving therapy with negative final margin status in primary breast cancer. Eur J Surg Oncol 2018; 44(9):13181323.

7. Chagpar AB, Tsangaris TN, Lannin DR. Do All Positive Margins in Breast Cancer Patients Undergoing a Partial Mastectomy Need to Be Resected? J Am Coll Surg 2018; 227(1):1321. 
8. Heelan Gladden AA, Sams S, Gleisner A, et al. Re-excision rates after breast conserving surgery following the 2014 SSO-ASTRO guidelines. Am J Surg 2017; 214(6):1104-1109.

9. Vos EL, Gaal J, Verhoef C, Brouwer K, van Deurzen CHM, Koppert LB. Focally positive margins in breast conserving surgery: Predictors, residual disease, and local recurrence. Eur J Surg Oncol 2017; 43(10):1846-1854.

10. Landercasper J, Attai D, Atisha D, et al. Toolbox to Reduce Lumpectomy Reoperations and Improve Cosmetic Outcome in Breast Cancer Patients: The American Society of Breast Surgeons Consensus Conference. Ann Surg Oncol 2015; 22(10):3174-83.

11. Grant Y, Al-Khudairi R, St John E, et al. Patient-level costs in margin re-excision for breastconserving surgery. Br J Surg 2019; 106(4):384-394.

12. Wood WC. Close/positive margins after breast-conserving therapy: additional resection or no resection? Breast 2013; 22 Suppl 2:S115-7.

13. Brouwer de Koning SG, Vrancken Peeters MTFD, Jóźwiak K, Bhairosing PA, Ruers TJM. Tumor Resection Margin Definitions in Breast-Conserving Surgery: Systematic Review and Meta-analysis of the Current Literature. Clin Breast Cancer 2018; 18(4):e595-e600.

14. Findlay-Shirras LJ, Outbih O, Muzyka CN, Galloway K, Hebbard PC, Nashed M. Predictors of Residual Disease After Breast Conservation Surgery. Ann Surg Oncol 2018; 25(7):19361942.

15. Tang R, Coopey SB, Specht MC, et al. Lumpectomy specimen margins are not reliable in predicting residual disease in breast conserving surgery. Am J Surg 2015; 210(1):93-8.

16. Zaidi M, Khan S, Farooqi NB, Abbas K, Idrees R. Effect of formalin fixation on surgical margins in breast cancer surgical specimen. Int J Breast Cancer 2014; 2014:121838.

17. Yeap BH, Muniandy S, Lee SK, Sabaratnam S, Singh M. Specimen shrinkage and its influence on margin assessment in breast cancer. Asian J Surg 2007; 30(3):183-7. 
18. Vos EL, Siesling S, Baaijens MHA, Verhoef C, Jager A, Voogd AC, Koppert LB. Omitting re-excision for focally positive margins after breast-conserving surgery does not impair disease-free and overall survival. Breast Cancer Res Treat 2017; 164(1):157-167.

19. McIntosh A, Freedman G, Eisenberg D, Anderson P. Recurrence rates and analysis of close or positive margins in patients treated without re-excisionbefore radiation for breast cancer. Am J Clin Oncol. 2007; 30(2):146-51.

20. Zhou M, Peddi P, Chu QD. Radiation Therapy for Positive Surgical Margins in Women $\geq 70$ Years with Stage I, Estrogen Receptor-positive Breast Cancer. Anticancer Res 2018; 38(9):5253-5260.

21. Park S, Ahn SD, Choi EK, Kim SS. The effect of escalating the boost dose for patients with involved resection margin after breast-conserving surgery. Jpn J Clin Oncol 2018; 48(3):272-277.

22. Houssami N, Macaskill P, Marinovich ML, Morrow $\quad$ M. The association of surgical margins and local recurrence in women with early-stage invasive breast cancer treated with breast-conserving therapy: a meta-analysis. Ann Surg Oncol 2014; 21(3):71730.

23. Mihalcik SA, Rawal B, Braunstein LZ, et al. The Impact of Reexcision and Residual Disease on Local Recurrence Following Breast-Conserving Therapy. Ann Surg Oncol 2017; 24(7):1868-1873. 


\section{Tables}

Table 1. Distribution of baseline variables after propensity score matching

\begin{tabular}{|c|c|c|c|}
\hline & $\begin{array}{c}\text { Cancer-free margins } \\
\qquad(\mathbf{n}=\mathbf{5 2 0})\end{array}$ & $\begin{array}{l}\text { Not re-excised } \\
\quad(n=104)\end{array}$ & p-value \\
\hline Age at diagnosis (years) & $63.3( \pm 12.6)$ & $63.2( \pm 13.6)$ & 0.79 \\
\hline \multicolumn{4}{|l|}{ Histological Type } \\
\hline DCIS & $91(17.5 \%)$ & $20(19.2 \%)$ & \\
\hline Ductal invasive carcinoma & $381(73.3 \%)$ & $70(67.3 \%)$ & 0.34 \\
\hline Lobular invasive carcinoma & $48(9.2 \%)$ & $14(13.5 \%)$ & \\
\hline \multicolumn{4}{|l|}{ Grading } \\
\hline G1 & $51(9.8 \%)$ & $7(6.7 \%)$ & \\
\hline G2 & $315(60.6 \%)$ & $66(63.5 \%)$ & 0.61 \\
\hline G3 & $154(29.6 \%)$ & $31(29.8 \%)$ & \\
\hline \multicolumn{4}{|l|}{ pT stage } \\
\hline pT1a/pT1mic & $22(4.2 \%)$ & $7(6.7 \%)$ & \\
\hline pT1b & $109(21.0 \%)$ & $22(21.2 \%)$ & \\
\hline pT1c & $222(42.7 \%)$ & $46(44.2 \%)$ & 0.52 \\
\hline pT2 & $76(14.6 \%)$ & $9(8.7 \%)$ & \\
\hline pTis & $91(17.5 \%)$ & $20(19.2 \%)$ & \\
\hline \multicolumn{4}{|l|}{ pN stage } \\
\hline $\mathrm{pN} 0 / \mathrm{pNmic}$ & $388(74.6 \%)$ & $78(75.0 \%)$ & \\
\hline pN1 & $92(17.7 \%)$ & $16(15.4 \%)$ & 0.72 \\
\hline pN2-3 & $40(7.7 \%)$ & $10(9.6 \%)$ & \\
\hline
\end{tabular}

Biomolecular subtype

Luminal A

$317(70.0 \%) \quad 62(59.6 \%)$

Luminal B

$152(29.2 \%)$

$27(26.0 \%)$

HER 2+

$9(1.7 \%)$

$6(5.8 \%)$

0.10

TNBC

$42(8.1 \%)$

$9(8.6 \%)$

Hormone therapy

No

$115(22.1 \%) \quad 28(26.9 \%)$

Yes

$405(77.9 \%)$

$76(73.1 \%)$

Chemotherapy

No

$409(78.6 \%) \quad 81(77.9 \%)$

Yes

$111(21.3 \%)$

$23(22.1 \%)$

0.86

\section{Radiation therapy}


No

Yes

Radiation boost

No

Yes
$97(18.6 \%)$

$423(81.4 \%)$

$41(9.7 \%)$

$382(90.3 \%)$
$11(10.6 \%)$

$93(89.4 \%)$

$4(4.3 \%)$

0.11

$89(95.7 \%)$ 
Table 2. Long-term outcomes between not re-excised vs. control patients

\begin{tabular}{lccc}
\hline & $\begin{array}{c}\text { Cancer-free margins } \\
(\mathbf{n}=\mathbf{5 2 0})\end{array}$ & $\begin{array}{c}\text { Not re-excised } \\
(\mathbf{n = 1 0 4})\end{array}$ & p-value \\
\hline Mean follow up (months) & $44.8( \pm 36.8)$ & $48.6( \pm 30.6)$ & 0.09 \\
Loco-regional recurrence & $491(94.4 \%)$ & $99(95.2 \%)$ & \\
No & $29(5.6 \%)$ & $5(4.8 \%)$ & \\
Yes & & & \\
Type of loco-regional recurrence & $19(65.5 \%)$ & $4(80.0 \%)$ & \\
In-breast local recurrence & $10(34.5 \%)$ & $1(20.0 \%)$ & \\
Axillary/Supra-clavicular nodal disease & & $102(98.1 \%)$ & \\
Distant metastases & $491(94.4 \%)$ & $2(1.9 \%)$ & \\
No & $29(5.6 \%)$ & & 0.12 \\
Yes & & $96(92.3 \%)$ & \\
Any first event & $462(88.9 \%)$ & $8(7.7 \%)$ & \\
No & $58(11.1 \%)$ & $100(96.1 \%)$ & \\
Yes & & $4(3.9 \%)$ & \\
Cancer-related death & $500(96.1 \%)$ & $20(3.9 \%)$ & \\
No & & \\
Yes & & & \\
\hline
\end{tabular}


Table 3. Multivariate analysis of clinical variables associated with loco-regional recurrence and distant metastases

\begin{tabular}{|c|c|c|c|c|c|c|}
\hline & \multicolumn{3}{|c|}{ Loco-regional recurrence } & \multicolumn{3}{|c|}{ Distant Metastases } \\
\hline & $\begin{array}{c}\text { Hazard } \\
\text { Ratio }\end{array}$ & $95 \% \mathrm{CI}$ & p-value & $\begin{array}{c}\text { Hazard } \\
\text { Ratio }\end{array}$ & $95 \% \mathrm{CI}$ & p-value \\
\hline Age at diagnosis & 1.03 & $(0.99-1.07)$ & 0.08 & 1.03 & $(0.99-1.07)$ & 0.12 \\
\hline \multicolumn{7}{|l|}{ Margins } \\
\hline Positive not re-excised vs. clear & 0.98 & $(0.36-2.67)$ & 0.96 & 0.37 & $(0.08-1.61)$ & 0.18 \\
\hline \multicolumn{7}{|l|}{ Histological type } \\
\hline Invasive ductal vs. DCIS & 2.09 & $(0.01-428.14)$ & 0.79 & 1.18 & $(0.0-7,165.96)$ & 0.97 \\
\hline Invasive lobular vs. DCIS & 2.77 & $(0.01-634.2)$ & 0.71 & 2.20 & $(0.0-13,874.6)$ & 0.86 \\
\hline \multicolumn{7}{|l|}{ pT stage } \\
\hline pT1 vs. pTis & 1.10 & $(0.01-225.0)$ & 0.92 & 1.35 & $(0.0-8,125.8)$ & 0.95 \\
\hline pT2-3 vs. pTis & 1.33 & $(0.01-300.18)$ & 0.92 & 2.36 & $(0.0-14,725.9)$ & 0.85 \\
\hline \multicolumn{7}{|l|}{ pN stage } \\
\hline pN0 vs. pN2 & 1.28 & $(0.24-6.73)$ & 0.77 & 0.37 & $(0.14-0.98)$ & 0.046 \\
\hline pN1 vs. pN2 & 0.72 & $(0.12-4.31)$ & 0.72 & 0.22 & $(0.07-0.72)$ & 0.013 \\
\hline \multicolumn{7}{|l|}{ Grading } \\
\hline G1 vs. G3 & 0.59 & $(0.13-2.64)$ & 0.49 & 2.43 & $(0.46-12.9)$ & 0.30 \\
\hline G2 vs. G3 & 0.57 & $(0.21-1.58)$ & 0.28 & 1.72 & $(0.71-4.17)$ & 0.23 \\
\hline \multicolumn{7}{|l|}{ Biomolecular subtype } \\
\hline Luminal A vs. TNBC & 0.63 & $(0.12-3.40)$ & 0.59 & 0.65 & $(0.15-2.81)$ & 0.56 \\
\hline Luminal B vs. TNBC & 0.65 & $(0.14-3.08)$ & 0.58 & 1.10 & $(0.32-3.77)$ & 0.88 \\
\hline HER2+ vs. TNBC & 0.34 & $(0.04-4.14)$ & 0.43 & NA* & NA & NA \\
\hline \multicolumn{7}{|l|}{ Chemotherapy } \\
\hline Yes vs. no & 0.52 & $(0.14-1.93)$ & 0.33 & 6.12 & $(1.97-18.99)$ & 0.002 \\
\hline \multicolumn{7}{|l|}{ Hormone therapy } \\
\hline Yes vs. no & 0.51 & $(0.19-1.41)$ & 0.20 & 0.50 & $(0.18-1.40)$ & 0.19 \\
\hline \multicolumn{7}{|l|}{ Radiation therapy } \\
\hline Yes vs. no & 0.48 & $(0.19-1.23)$ & 0.13 & 1.07 & $(0.27-4.20)$ & 0.92 \\
\hline
\end{tabular}

*NA: not available, due to lack of patients in one category. 


\section{Figure Captions}

Fig. 1 Survival probability curves of patients with positive margins not undergoing re-excision vs. patients with clear margins after lumpectomy. a) 5-years loco-regional recurrence-free survival probability; b) 5-years distant metastases-free survival probability. 